Research, Society and Development, v. 11, n. 2, e34011225818, 2022

(CC BY 4.0) | ISSN 2525-3409 | DOI: http://dx.doi.org/10.33448/rsd-v11i2.25818

\title{
O atendimento interdisciplinar na Atenção Primária em Saúde - Revisão integrativa
}

\author{
Interdisciplinary care in Primary Health care - Integrative review
}

Atención interdisciplinaria en la Atención Primaria de Salud - Revisión integradora

Patricia Cortez de Medeiros ORCID: https://orcid.org/0000-0003-1420-084X Centro Universitário Maurício de Nassau, Brasil

E-mail: patyycortez@yahoo.com.br

Graziely Veríssimo de Melo

ORCID: https://orcid.org/0000-0002-1694-1791 Centro Universitário Maurício de Nassau, Brasil

E-mail: grazielyvm16@gmail.com

Artur Vinicius Lima e Silva

ORCID: https://orcid.org/0000-0002-6970-4167

Universidade de Pernambuco, Brasil

E-mail: arturviniciuslima@gmail.com

Ruth Elisa de Lima Freitas

ORCID: https://orcid.org/0000-0002-2413-8747

Universidade de Pernambuco, Brasil

E-mail: arturviniciuslima@gmail.com

Brunna Caroline Santos de Moura ORCID: https://orcid.org/0000-0001-5253-4352

Universidade de Pernambuco, Brasil

E-mail: brunnacsmoura@gmal.com

Halanderson Cabral da Costa

ORCID: https://orcid.org/0000-0002-1982-5120

Centro Universitário UNIBRA, Brasil

E-mail: halan.costaa@gmail.com

Alyce Oliveira da Silva

ORCID: https://orcid.org/0000-0002-8728-6210

Centro Universitário Maurício de Nassau, Brasil

E-mail: alyce__hotmail.com

Elton de Oliveira Coutinho

ORCID: https://orcid.org/0000-0003-0040-6548

Centro Universitário Maurício de Nassau, Brasil

E-mail: elton.coutinho44@gmail.com

Jafia Andreza Gonçalves de Oliveira

ORCID: https://orcid.org/0000-0001-9028-0963

Centro Universitário Maurício de Nassau, Brasil

E-mail: jafiaandreza@hotmail.com

Carlos Henrique Souza Andrade

ORCID: https://orcid.org/0000-0003-2545-2428

Centro Universitário São Miguel, Brasil

E-mail: Henrique.enffsm@hotmail.com

Raylena de Andrade Catunda da Silva ORCID: https://orcid.org/0000-0002-5924-1306

Centro Universitário Unit, Brasil

E-mail: ray_catunda@hotmail.com

Rianne Milenne de Lima Moraes Santos

ORCID: https://orcid.org/0000-0002-7077-906X Centro Universitário Maurício de Nassau, Brasil

E-mail: riannefer123@gmail.com

Rivaldo Alexsandro da Silva

ORCID: https://orcid.org/0000-0002-7342-9604

Universidade Estácio de Sá Brasil

E-mail: rivaldoenf@gmail.com

Sheila Absalão Azevedo

ORCID: https://orcid.org/0000-0002-6970-4167

Centro Universitário Maurício de Nassau, Brasil

E-mail: Sheila_absalao@hotmail.com 


\begin{abstract}
Resumo
O desempenho dos profissionais de saúde em equipes interdisciplinares na atenção primária em saúde do Sistema único de Saúde ganhou destaque no Brasil, principalmente durante a pandemia do COVID-19, época em que a saúde pública no país entrou em colapso, e que houve a necessidade de um maior número de profissionais atuando na área. Nesse sentido, o objetivo desse estudo foi avaliar o reflexo que a participação interdisciplinar possui na Atenção Primária (APS). Foram realizadas buscas nas bases de dados Scielo, Scopus, BVS, e no Google Acadêmico. Foram encontrados 285 artigos, dos quais, 44 apresentaram texto completo, sendo analisados seus resumos. Logo em seguida, 13 textos foram selecionados para análise. Observou-se que nos artigos investigados o trabalho em equipe tem contribuído efetivamente na evolução dos pacientes, e viabilizou a organização da atenção em saúde em uma perspectiva de práticas clínicas ampliadas centralizadas no indivíduo, na família e comunidade.
\end{abstract}

Palavras-chave: Saúde pública; Atenção primária em saúde; Sistema Único de Saúde.

\begin{abstract}
The performance of health professionals in interdisciplinary teams in primary health care of the Unified Health System gained prominence in Brazil, especially during the COVID-19 pandemic, a time when public health in the country collapsed, and there was a need of a greater number of professionals working in the area. In this sense, the objective of this study was to evaluate the impact that interdisciplinary participation had on care provided by health teams and on the clinical evolution of patients. Searches were carried out in the Virtual Health Library - VHL, which includes PubMed, SciELO and Lilacs, and in Google Scholar. A total of 285 articles were found, of which 44presented full text, and their abstracts were analyzed. Soon after, 13 texts were selected for analysis. It was observed that, in the articles investigated, teamwork has effectively contributed to the evolution of patients, and enabled the organization of health care in a perspective of expanded clinical practices centered on the individual, family and community.
\end{abstract}

Keywords: Public health; Primary health care; Health Unic System.

\title{
Resumen
}

La actuación de los profesionales de la salud en equipos interdisciplinarios en la atención primaria de salud del Sistema Único de Salud ganó destaque en Brasil, especialmente durante la pandemia de la COVID-19, momento en que la salud pública en el país colapsó y hubo necesidad de un mayor número de profesionales que trabajan en el área. En ese sentido, el objetivo de este estudio fue evaluar el impacto que la participación interdisciplinaria tuvo en la atención brindada por los equipos de salud y en la evolución clínica de los pacientes. Las búsquedas fueron realizadas en la Biblioteca Virtual en Salud - BVS, que incluye PubMed, SciELO y Lilacs, y en Google Scholar. Se encontraron un total de 285 artículos, de los cuales 244 presentaban texto completo, y se analizaron sus resúmenes. Poco después, se seleccionaron 13 textos para su análisis. Se observó que en los artículos investigados, el trabajo en equipo ha contribuido efectivamente para la evolución de los pacientes y viabilizó la organización de la atención a la salud en una perspectiva de prácticas clínicas ampliadas centradas en el individuo, la familia y la comunidad.

Palabras clave: Salud pública; Primeros auxilios; Sistema Único de Salud.

\section{Introdução}

A atenção primária à saúde (APS) tem função centralizadora na organização dos serviços de saúde no SUS. Criada em 1996, a APS é implementada no país através da estratégia de saúde da família (ESF), que determina um modelo centrado de APS baseado na coordenação do cuidado e respeitando os princípios da integralidade, universalidade e equidade previstos no SUS, tendo o trabalho em equipe como uma de suas diretrizes operacionais (Geremia,2020).

Contudo, as necessidades no âmbito da saúde durante a pandemia do Covid-19 estão cada vez mais desafiadoras, o que impactou a resolutividade dos serviços oferecidos no Sistema único de Saúde (SUS). Em contrapartida, as complexidades apresentadas principalmente pela excessiva carga de trabalho, e a necessidade um número cada vez maior de profissionais da saúde atuando contra o vírus ocorreram nos três níveis de atenção, mas principalmente na atenção primária pois esta é a porta de entrada do SUS. (Geremia, 2020). Nesse sentido, o atendimento interdisciplinar contribuiu para desmistificar e viabilizar uma nova forma de organização na atenção primária, visando a melhoria das práticas clínicas e centralizando a atenção nas necessidades do indíviduo. (Silva, 2015)

$\mathrm{O}$ atendimento interdisciplinar acontece quando diversos profissionais de saúde, de diversas áreas, como: enfermagem, medicina, odontologia, fisioterapia, entre outros, se unem afim de trabalharem em equipe, cujo objetivo é o 
planejamento das ações e a implementação destas na unidade de saúde nos quais desenvolvem suas atividades. Desta forma, os profissionais devem atuar de forma reciproca e solidária, respeitando as normas e tudo que lhe é proposto afim de práticas cada vez mais inclusivas com os os usuários. (Reeves et al, 2018).

Dessa forma a conjuntura interdisciplinar, mantém relações sociais horizontais, o que inspira um trabalho integrado e completo pois integra diversas áreas do saber, e resulta na compreensão da mensagem que o paciente emite. Nessa perspectiva, as equipes multiprofissionais de saúde devem: agir integrando as diversas competências e categorias profissionais que as compõem; primar pela ação articulada com setores que as cercam com base nos determinantes socioculturais do processo saúde-doença, fugindo da lógica do atendimento curativo; ter foco na horizontalidade, vínculo e corresponsabilidade pelas ações junto aos usuários do sistema (Moretti, 2009).

\section{Metodologia}

Trata-se de uma revisão integrativa com abordagem qualitativa de natureza exploratória, que consiste em um método de pesquisa da prática baseada em evidências, pois sintetiza as pesquisas disponíveis sobre determinado tema, fundamentandose em conhecimento científico (Souza et al; 2010). O processo de elaboração do presente estudo foi realizado entre novembro e dezembro de 2021 e apresentou as seguintes fases: (i) definição da pergunta norteadora, estratégia de busca na literatura, identificação dos estudos e coleta de dados; (ii) análise dos estudos incluídos; (iii) discussão dos resultados (iiii) apresentação da revisão integrativa.

\section{Definição da pergunta norteadora}

Qual é o reflexo do atendimento interdisciplinar na atenção primária em saúde?

\section{Estratégia de busca na literatura}

Realizou-se um levantamento de publicações em quatro bases de dados: 1. Scielo 2. Scopus; 3. BVS e 4. Google acadêmico. A pesquisa envolveu as seguintes combinações de palavras-chave: (a) Atenção Primária em Saúde "and" Atendimento interdisciplinar; (b) Atendimento multiprofissional.

\section{Critérios de inclusão}

As publicações resultantes da busca nas bases de dados foram selecionadas a partir dos seguintes critérios de inclusão:

- Publicações disponibilizadas nas línguas portuguesa, inglesa ou espanhola;

- Disponibilidade dos textos na versão integral;

- Período de publicação entre 2015 e 2021;

- Busca por artigos científicos, excluindo-se outros tipos de trabalhos (teses, dissertações, livros e resenhas).

\section{Critérios de exclusão}

Os critérios de exclusão foram as publicações em duplicidade nas diferentes bases de dados e aquelas em que o objeto de estudo não estabelecia relação com o tema abordado.

\section{Identificação dos estudos e coleta de dados}

Inicialmente todos os estudos identificados na pesquisa foram avaliados a partir de seus títulos e/ou resumos. Posteriormente, os estudos que atenderam aos critérios de inclusão foram recuperados para leitura do texto completo e nova 
avaliação quanto aos critérios de inclusão. Do total de estudos selecionados, foi feita uma leitura prévia e identificados alguns eixos temáticos para análise. Após essa etapa, as informações extraídas dos estudos selecionados incluíram: título, ano de publicação, idioma, o objeto do estudo e base de dados onde foi publicado. A Figura 1 apresenta o fluxograma desenvolvido para atender o objetivo do estudo.

Figura 1 - Fluxograma do processo metodológico de busca e análise dos estudos.

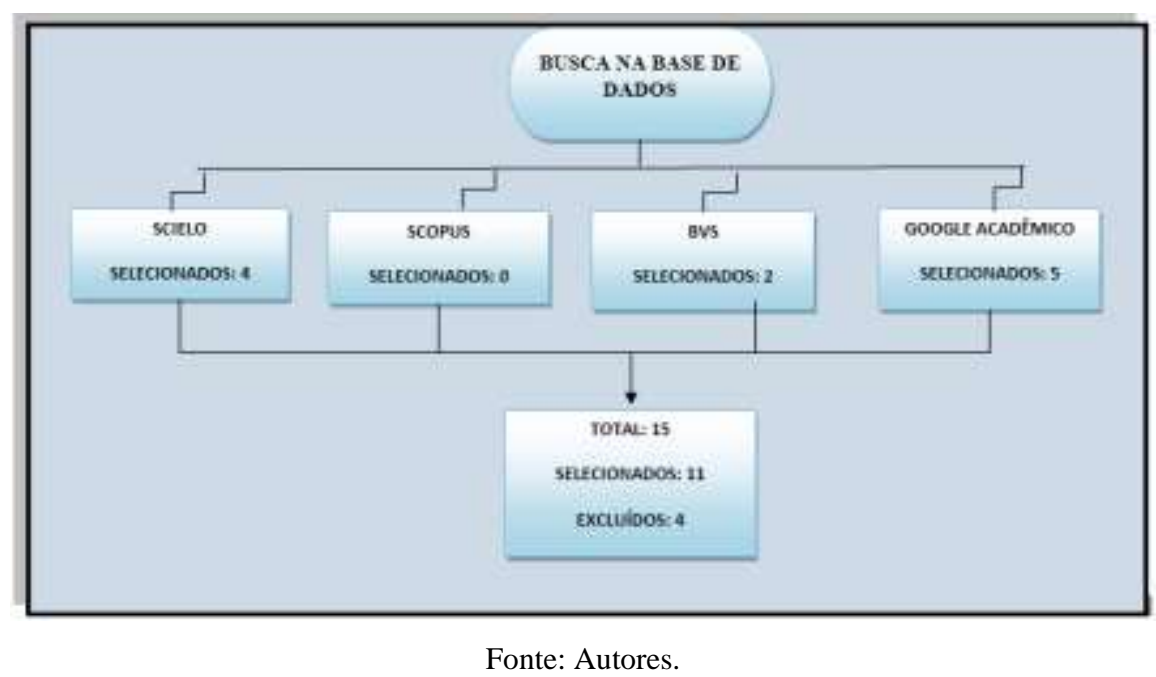

\section{Discussão dos resultados}

A discussão dos resultados foi realizada de forma exploratória e comparativa, a partir da interpretação e síntese dos autores abordados, baseando-se na literatura científica do assunto e visando atender ao objetivo proposto do estudo.

\section{Resultados e Discussão}

Foram selecionados onze artigos para análise, sendo 04 da base de dados SCIELO, 02 da base de dados BVS e 05 da base de dados do Google Acadêmico. Com relação a escrita, todos estão redigidos na língua portuguesa e publicados nos últimos dez anos.

Em relação ao Quadro 1, tem-se a seguir uma sinopse que contém as principais características dos artigos selecionados neste trabalho, sendo os mesmos retratados com título do estudo, autor, ano de publicação e objetivo. 
Quadro 1- Sinopse demonstrativa dos artigos incluídos no estudo.

\begin{tabular}{|c|c|c|c|c|}
\hline $\mathbf{N}^{\circ}$ & Título & Autores & Ano & Objetivo \\
\hline 1 & $\begin{array}{l}\text { Acolhimento na Atenção Primária à } \\
\text { Saúde: revisão integrativa }\end{array}$ & $\begin{array}{l}\text { Larissa Rachel Palhares } \\
\text { Coutinho, Ana Rita } \\
\text { Barbieri, Mara Lisiane } \\
\text { de Moraes dos Santos }\end{array}$ & 2015 & $\begin{array}{l}\text { Analisar o conhecimento produzido sobre o } \\
\text { acolhimento na Atenção Primária à Saúde, nos } \\
\text { últimos oito anos }\end{array}$ \\
\hline 2 & $\begin{array}{l}\text { Atenção centrada no paciente na } \\
\text { prática interprofissional colaborativa }\end{array}$ & $\begin{array}{l}\text { Heloise Fernandes } \\
\text { Agreli,Marina Peduzzi, } \\
\text { e Mariana Charantola } \\
\text { Silva }\end{array}$ & 2016 & $\begin{array}{l}\text { Descrever os elementos-chave da atenção centrada } \\
\text { no paciente e sua relação com a prática } \\
\text { interprofissional colaborativa na atenção primária à } \\
\text { saúde, no contexto do Sistema Único de Saúde, }\end{array}$ \\
\hline 3 & $\begin{array}{l}\text { Atitudes para a colaboração } \\
\text { interprofissional de equipes da } \\
\text { Atenção Primária participantes do } \\
\text { Programa Mais Médicos }\end{array}$ & $\begin{array}{l}\text { José Rodrigues Freire } \\
\text { Filho Marcelo Viana da } \\
\text { Costa Carinne } \\
\text { Magnago, e Aldaísa } \\
\text { Cassanho Forster }\end{array}$ & 2018 & $\begin{array}{l}\text { Comparar atitudes em relação à colaboração } \\
\text { interprofissional de profissionais de saúde } \\
\text { componentes de equipes da Estratégia Saúde da } \\
\text { Família. }\end{array}$ \\
\hline 4 & $\begin{array}{l}\text { Atuação em } \quad \text { equipes } \\
\text { multiprofissionais de saúde: uma } \\
\text { revisão sistemática }\end{array}$ & $\begin{array}{l}\text { Patrícia Andréia da } \\
\text { Silva, et al. }\end{array}$ & 2013 & $\begin{array}{l}\text { Investigar a produção científica relacionada à } \\
\text { atuação em equipes multiprofissionais de saúde nos } \\
\text { últimos dez anos }\end{array}$ \\
\hline 5 & $\begin{array}{l}\text { Coordenação do cuidado e Atenção } \\
\text { Primária à Saúde no Sistema Único } \\
\text { de Saúde }\end{array}$ & $\begin{array}{l}\text { Patty Fidelis de } \\
\text { Almeida, et al. }\end{array}$ & 2018 & $\begin{array}{l}\text { A identificação de políticas, estratégias e } \\
\text { instrumentos para alcance de melhor coordenação } \\
\text { no Sistema Único de Saúde }\end{array}$ \\
\hline 6 & $\begin{array}{l}\text { Facilidades e } \\
\text { implementação dificuldades para } \\
\text { interprofissional na atenção primária } \\
\text { à saúde: revisão integrativa }\end{array}$ & $\begin{array}{l}\text { Tayná Martins de } \\
\text { Medeiros, et al. }\end{array}$ & 2021 & $\begin{array}{l}\text { Identificar as facilidades e dificuldades para } \\
\text { implementação da educação interprofissional na } \\
\text { atenção básica em saúde }\end{array}$ \\
\hline 7 & $\begin{array}{l}\text { Interprofissionalidade na atenção } \\
\text { primária e a saúde da pessoa idosa: } \\
\text { revisão integrativa }\end{array}$ & $\begin{array}{l}\text { Lucenilde Rodrigues } \\
\text { Carvalho \& Valéria de } \\
\text { Sousa Queiroz }\end{array}$ & 2021 & $\begin{array}{l}\text { Descrever a interprofissionalidade e as práticas } \\
\text { colaborativas na Atenção Primária à Saúde (APS), e } \\
\text { as contribuições para o enfermeiro no cuidado à } \\
\text { saúde da pessoa idosa }\end{array}$ \\
\hline 8 & $\begin{array}{l}\text { O desafio e potencialidade da } \\
\text { interdisciplinaridade no atendimento } \\
\text { à saúde }\end{array}$ & $\begin{array}{l}\text { Horst, Viviane Silveira } \\
\text { Batista; Orzechowski, } \\
\text { Suzete Terezinha }\end{array}$ & 2017 & $\begin{array}{l}\text { Discutir o atendimento interdisciplinar na área da } \\
\text { saúde no Brasil }\end{array}$ \\
\hline 9 & $\begin{array}{l}\text { Integração entre currículos na } \\
\text { educação de profissionais da Saúde: a } \\
\text { potência para educação } \\
\text { interprofissional na graduação }\end{array}$ & $\begin{array}{lr}\text { Luciane } & \text { Ines } \\
\text { Ramona } & \text { Fly(a) } \\
\text { Cernanda } \\
\text { Ceriotti Toassi(b) }\end{array}$ & 2018 & $\begin{array}{l}\text { Se propôs a compreender os significados da } \\
\text { vivência multiprofissional na atividade de ensino } \\
\text { integradora de uma universidade pública do sul do } \\
\text { Brasil, que acontece em serviços da Atenção } \\
\text { Primária à Saúde, analisando seu potencial para a } \\
\text { educação interprofissional (EIP). }\end{array}$ \\
\hline 10 & $\begin{array}{l}\text { Interprofissionalidade e saúde mental: } \\
\text { uma revisão integrativa }\end{array}$ & $\begin{array}{lr}\text { Estefânia } & \text { Bisognin } \\
\text { Cervo1 } & \text { Malwiani } \\
\text { Aparecida } & \text { Caumo Ana } \\
\text { Luiza } & \text { Parcianello } \\
\text { Cerdótes } & \text { Fernanda Pires } \\
\text { Jaeger } & \end{array}$ & 2020 & $\begin{array}{l}\text { Analisar as pesquisas científicas sobre o trabalho } \\
\text { interprofissional na saúde mental. }\end{array}$ \\
\hline
\end{tabular}

Fonte: Autores.

$\mathrm{O}$ atendimento interdisciplinar evidencia um atendimento integrado e, mas no indivíduo como um todo proporcionando uma melhor qualidade de vida. Desta forma, a atenção interdisciplinar não seria resultado de uma simples síntese, mas de sínteses imaginativas e audazes; interdisciplinaridade não é categoria de conhecimento, mas de ação; a 
Research, Society and Development, v. 11, n. 2, e34011225818, 2022

(CC BY 4.0) | ISSN 2525-3409 | DOI: http://dx.doi.org/10.33448/rsd-v11i2.25818

interdisciplinaridade nos conduz a um exercício de conhecimento: o perguntar e o duvidar; entre as disciplinas e a interdisciplinaridade existe uma diferença de categoria. (Fazenda, 2012).

A Organização Mundial da Saúde reconhece a colaboração interprofissional como uma estratégia inovadora na redução da crise de saúde mundial. A educação interprofissional e a prática colaborativa podem ser mal compreendidas pelos profissionais de saúde, ao interpretarem simplesmente como trabalhar junto uns com os outros. Colaboração, se refere à criação de sinergia e não somente a acordo e comunicação e o trabalho colaborativo dos profissionais de saúde pode impactar sobre os desafios atuais de saúde, fortalecer o sistema e imprimir melhorias de resultados na saúde (OMS, 2010).

Para que o atendimento interdisciplinar aconteça na atenção básica, há a necessidade de se desenvolver uma metodologia que integre mais de uma categoria de profissionais de saúde como: psicólogos, nutricionistas, médicos, enfermeiros, terapeutas ocupacionais, assistentes sociais, fisioterapeutas, pedagogos, odontólogos, entre outros, conforme a necessidade de cada região. O objetivo principal é proporcionar ao paciente um atendimento de qualidade, realizado por um conjunto de profissionais, os quais deverão se unir ao propor uma solução em conjunto para o respectivo problema apresentado pelo paciente, bem como levantado pela equipe de profissionais desde o acolhimento do paciente, por meio de um olhar multiprofissional e interdisciplinar, que somente acrescentará ao plano de tratamento de cada paciente, segundo necessidade. (Gomes, 1997)

No Brasil, o termo equivalente à Atenção Primária em Saúde é a Atenção Básica (AB). Estes termos são associados às noções de vinculação e responsabilização, acessibilidade, atenção abrangente e integral, resolutividade e protagonismo na gestão do cuidado (Brasil, 2011).

O modelo utilizado no Brasil, orientado na APS, é a Estratégia Saúde da Família (ESF), que teve início em 1994 e foi idealizado, inicialmente, como uma ferramenta de extensão da cobertura assistencial. Atualmente, é o maior programa assistencial no País e é considerado como um reorganizador do Sistema Único de Saúde (SUS), carregando enorme potencial para estruturar de forma consistente a APS no Brasil. Tem sido apontado, por sua extensão e cobertura, como um modelo a ser seguido (Abrahão, 2007; \&Mendes, 2013).

A incorporação do atendimento interdisciplinar na atenção à saúde se fez necessário devido a alguns aspectos da realidade de produção dos serviços que se configura por forte fragmentação do trabalho em saúde, exposição do usuário/paciente a atos duplicados, maiores possibilidades de erros de diagnóstico e tratamento, bem como reduzida capacidade resolutiva e aumento dos custos (Costa, 2019). Um dos pontos divergentes e que contribuem para os problemas enfrentados no sistema de trabalho refere-se a profissionais de saúde trabalharem juntos, mas serem formados separadamente (Costa, 2019).

Além da comunicação, o trabalho em equipe é dificultado na atenção básica devia a fatores externos e internos que colaboram para uma relação social deficiente e que foi construída por relações de hierarquias falhas, e pela desvalorização de algumas categorias profissionais, além de fortes influências políticas que afetam a prática colaborativa entre os trabalhadores de saúde sob um viés negativo (Freire Filho et al., 2018). O trabalho em equipe é algo extremamente importante, pois, além de se configurar como essencial para o enfrentamento das necessidades de saúde, requer uma abordagem mais ampliada e organizada por parte dos serviços. Além disso, a articulação conjunta dos processos de trabalho em saúde permite consolidar uma nova concepção de cuidado, horizontalizando a prestação de uma assistência mais qualificada e integral (Peduzzi et al., 2020).

Sendo assim, a atuação uniprofissional ocasiona segregação dos profissionais de saúde, prejudicando a articulação do processo. A saúde-doença, enquanto práticas interprofissionais promovem a colaboração entre os diferentes profissionais, possibilitando a união dos mesmos em torno do cuidado das necessidades de saúde do usuário ou comunidade (SILVA et al., 
Research, Society and Development, v. 11, n. 2, e34011225818, 2022

(CC BY 4.0) | ISSN 2525-3409 | DOI: http://dx.doi.org/10.33448/rsd-v11i2.25818

2015). O sucesso do trabalho colaborativo depende de fatores relacionados aos usuários e profissionais, fatores interpessoais, motivacionais, organizacionais e externos (Van Dongen et al., 2016).

Portanto, atualmente houve um avanço na discussão sobre a educação interprofissional que se destacou nacional e internacionalmente pela necessidade de reorganização dos serviços de saúde no contexto das diferentes demandas e do impacto sobre a saúde da população, principalmente após a pandemia do Covid-19. Tal reflexo serve para contribuir para a promoção de práticas de cuidado integral, com maior efetividade, resolutividade e segurança do paciente. A prática colaborativa entre os profissionais da saúde oportuniza o desenvolvimento de atitudes, habilidades e conhecimentos indispensáveis para a concretude do trabalho interprofissional em saúde (Reeves, 2016).

\section{Conclusão}

O presente estudo aponta que ainda há dificuldades de implementação de atividades interdisciplinares na rotina da atenção básica, já que ainda há equipes que são compostas apenas por médicos e enfermeiros. O que resulta na dificuldade de integração das categorias profissionais e reflete outras esferas da Rede de Atenção pois a APS é uma das portas de entrada do Sistema Único de Saúde. A falta de comunicação, e o excesso de trabalho principalmente durante a pandemia compactuou com a resistência de alguns profissionais de promover práticas colaborativas, o que deu ênfase a atividades uniprofissionais. Outra barreira importante, é a vigência do modelo biomédico, bem como a falta de recursos, capacitação profissional deficiente, e discordância de diagnóstico.

Sendo a Atenção Primária em Saúde ordenadora do SUS, é indispensável a necessidade de prática integrativas e colaborativas pois as atividades interdisciplinares tornam o atendimento mais completo, como apresenta uma melhor evolução clínica do paciente já que a necessidade deste é percebida como um todo, e cria oportunidade para que outros profissionais de saúde integrem a equipe e beneficiem a comunidade.

Portanto, é possível concluir que o trabalho em equipe é muito benéfico, como a troca de experiências entre o profissional e paciente, o respeito ao trabalho e à opinião de outros profissionais. Diante dos resultados, propõe-se uma valorização de atuação desses profissionais, visando a ampliar a discussão e aprimorar o trabalho em equipe de saúde, principalmente na Atenção Primária em Saúde.

\section{Referências}

Geremia, Daniela Savi (2020). Atenção Primária à Saúde em alerta: desafios da continuidade do modelo assistencial. Physis: Revista de Saúde Coletiva [online]. 30(1).

Brasil. Ministério da Saúde. (2020) Política Nacional de Atenção Básica, 2017. Brasília: MS.

Silva, J. A.M. et al (2015). Educação interprofissional e prática colaborativa na Atenção Primária à Saúde. Revista da Escola de Enfermagem da USP. 49(2), 16-24,. http:// dx.doi.org/10.1590/s0080-623420150000800003.

Reeves, S. et al. (2017). Interprofessional collaboration to improve professional practice and healthcare outcomes. Cochrane Database Of Systematic Reviews. 6(6), 2-50, http://dx.doi.org/10.1002/14651858.cd000072.pub3.

Moretti-Pires., Rodrigo Otávio e Campos \& Dalvan Antônio de. (2010) Equipe multiprofissional em Saúde da Família: do documental ao empírico no interior da Amazônia. Revista Brasileira de Educação Médica, 34(1). https://doi.org/10.1590/S0100-55022010000300007.

Fazenda, I. C. A. (2012) Interdisciplinaridade: história, teoria e pesquisa. (18 a ed.). Campinas, SP: Papirus.

Gomes, D. C. R., (2017) Interdisciplinaridade em saúde: um princípio a ser resgatado. Uberlândia: Edufu,.

Ministério da Saúde. (2011). Secretaria de Atenção à Saúde. Departamento de Atenção Básica. Acolhimento à demanda espontânea. Brasília: Ministério da Saúde.

Abrahão, A. L. (2007). Atenção primária e o processo de trabalho em saúde. Informe-se em promoção da saúde, Niterói, 3(1), 1-3.

Mendes, E. V., (2013) 25 anos do Sistema Único de Saúde: resultados e desafios. Estudos Avançados, São Paulo, 27(78), 27-34, 2013. 
Research, Society and Development, v. 11, n. 2, e34011225818, 2022

(CC BY 4.0) | ISSN 2525-3409 | DOI: http://dx.doi.org/10.33448/rsd-v11i2.25818

Freire Filho, J. R. et al., (2018). Attitudes towards interprofessional collaboration of Primary Care teams participating in the 'More Doctors' (Mais Médicos) program. Revista Latino-Americana de Enfermagem. 26, 1-8, ago. http://dx.doi.org/10.1590/1518-8345.2731.3018.

Bispo J., José Patrício.; Moreira \& Diane Costa. (2017). Educação permanente e apoio matricial: formação, vivências e práticas dos profissionais dos núcleos de apoio à saúde da família e das equipes apoiadas. Cadernos de Saúde Pública, [S.L.], 33(9), 1-13, 28 set. 2017. FapUNIFESP (SciELO). http://dx.doi.org/10.1590/0102-311x00108116.

Peduzzi, M. et al. (2020) Trabalho em equipe: uma revisita ao conceito e a seus desdobramentos no trabalho interprofissional. Trab. Educ. Saúde. 18(1), 1-20, https://doi. org/10.1590/1981-7746-sol00246

Peruzzo, H. E. et al.(2016). Os desafios de se trabalhar em equipe na Estratégia Saúde da Família. Escola Anna Nery. 22(4), 1-9, https://doi.org/10.1590/21779465-ean-2017- 0372

PReviato, G. F \&; BAldissera, V. D. A. (2018) Retratos da prática interprofissional colaborativa nas equipes da atenção primária à saúde. Revista Gaúcha de Enfermagem, 39, 1-9,http://dx.doi.org/10.1590/1983-1447.2018.2017-0132.

Reeves, S. et al(2017). Interprofessional collaboration to improve professional practice and healthcare outcomes. Cochrane Database Of Systematic Reviews. 6(6), 2-50,. http://dx.doi.org/10.1002/14651858.cd000072.pub3.

Van Dongen, J.J.J. et al.(2016). Interprofessional collaboration regarding patients' care plans in primary care: a focus group study into influential factors. Bmc Family Practice, [s. 1], 1(17), 58-68.

Tschannen, D.; Dorn, R.; \& Tadesco, C.(2018). Improving knowledge and behavior of leadership and followership among the interprofessional team. International Journal of Medical Education. 9, 82-188.

Pinheiro, G.E.W.; Azambuja, M.S.; Bonamigo \& Andrea W. (2017). Facilidades e dificuldades vivenciadas na Educação Permanente em Saúde, na Estratégia Saúde da Família. Saúde em Debate. 42(4), 187-197. http://dx.doi.org/10.1590/0103-11042018s415.

Agreli, H. F., Peduzzi, M. S. \& Mariana C., (2016). Atenção centrada no paciente na prática interprofissional colaborativa. Interface - Comunicação, Saúde, Educação, 20(59). 905-916. ISSN 1807-5762. https://doi.org/10.1590/1807-57622015.0511.

Freire, J. R. et al. (2018). Attitudes towards interprofessional collaboration of Primary Care teams participating in the 'More Doctors' (Mais Médicos) program. Revista Latino-Americana de Enfermagem, v. 26.

Almeida, Patty Fidelis de et al. (2018) Coordenação do cuidado e Atenção Primária à Saúde no Sistema Único de Saúde. Saúde em Debate, v. 42, n. spe1, pp. 244-260. <https://doi.org/10.1590/0103-11042018S116>. ISSN 2358-2898. https://doi.org/10.1590/0103-11042018S116.

Horst, V. \& Orzechowski, S. (2017). O Desafio e potencialidade da interdisciplinaridade no atendimento á saúde. Laplage em Revista. 3.192. $10.24115 /$ S2446-6220201731230p.192-201

Ely, L. I. T., \& Ramona F.C. (2018). Integração entre currículos na educação de profissionais da Saúde: a potência para educação interprofissional na graduação. Interface - Comunicação, Saúde, Educação v. 22 https://doi.org/10.1590/1807-57622017.0658.

Cervo, E. B.., Caumo, M. A., Parcianello Cerdótes , A. L., \& Pires Jaeger , . F. (2020). interprofissionalidade e saúde mental: uma revisão integrativa. Psicologia E Saúde Em Debate, 6(2), 260-272. https://doi.org/10.22289/2446-922X.V6N2A17. 